\title{
STRONG UNBOUNDEDNESS OF UNBOUNDED ANALYTIC FUNCTIONS
}

\author{
A. YA. GORDON
}

(Communicated by Albert Baernstein II)

\begin{abstract}
It is proved that if $f$ is an unbounded analytic function in the open unit disc $\mathbb{D}$, then there must exist a sequence $\left(z_{n}\right)$ in $\mathbb{D}$ such that for every $j=0,1,2, \ldots, f^{(j)}\left(z_{n}\right) \rightarrow \infty$ as $n \rightarrow \infty$. This answers affirmatively a question asked by J. Langley and L. Rubel in 1984.
\end{abstract}

One of the problems raised in [1] was the following: If $f(\cdot)$ is an unbounded analytic function in the open unit disc $\mathbb{D}$, must there exist a sequence $\left(z_{n}\right)$ in $\mathbb{D}$ such that for every $j=0,1,2, \ldots f^{(j)}\left(z_{n}\right) \rightarrow \infty$ as $n \rightarrow \infty$ ?

The answer is "yes", 1 and we give a simple proof.

Proof. By the hypothesis, there is a sequence $\left(z_{m}\right)$ in $\mathbb{D}=\{|z|<1\}$ such that $f\left(z_{m}\right) \rightarrow \infty$ as $m \rightarrow \infty$. Hence, to prove the theorem, it is enough to show that if $\left(z_{m}\right)$ is a sequence in $\mathbb{D}$ such that $f^{(j)}\left(z_{m}\right) \rightarrow \infty(m \rightarrow \infty)$ for $j=$ $0,1, \ldots, k$, then we can find a sequence $\left(z_{m}^{\prime}\right)$ in $\mathbb{D}$ such that $f^{(j)}\left(z_{m}^{\prime}\right) \rightarrow \infty$ $(m \rightarrow \infty)$ for $j=0,1, \ldots, k+1$. This latter result can be proved as follows.

Assume that $\left(z_{m}\right)$ is a sequence in $\mathbb{D}$ such that $f^{(j)}\left(z_{m}\right) \rightarrow \infty(m \rightarrow \infty)$ for $j=0,1, \ldots, k$. If $\left(f^{(k+1)}\left(z_{m}\right)\right)_{m=1}^{\infty}$ is unbounded, then there is a subsequence $\left(z_{m}^{\prime}\right)$ of $\left(z_{m}\right)$ such that $f^{(j)}\left(z_{m}^{\prime}\right) \rightarrow \infty(m \rightarrow \infty)$ for $j=0,1, \ldots, k+1$. So let $\left(f^{(k+1)}\left(z_{m}\right)\right)_{m=1}^{\infty}$ be bounded.

Split $f$ into the sum $f(z)=T_{m}(z)+R_{m}(z)$, where

$$
T_{m}(z)=f\left(z_{m}\right)+\frac{f^{\prime}\left(z_{m}\right)}{1 !}\left(z-z_{m}\right)+\cdots+\frac{f^{(k)}\left(z_{m}\right)}{k !}\left(z-z_{m}\right)^{k}
$$

is the Taylor polynomial of degree $k$ and

$$
R_{m}(z)=\frac{1}{k !} \int_{z_{m}}^{z} f^{(k+1)}(\zeta)(z-\zeta)^{k} d \zeta .
$$

Differentiation gives relations

$$
f^{(j)}(z)=T_{m}^{(j)}(z)+R_{m}^{(j)}(z)
$$

Received by the editors October 7, 1992 and, in revised form January 25, 1993.

1991 Mathematics Subject Classification. Primary 30D20.

${ }^{1}$ The authors of [1] proved this assertion under a certain growth condition on $f$, using Nevanlinna theory (see [2]). 
and

$$
R_{m}^{(j)}(z)=\frac{1}{(k-j) !} \int_{z_{m}}^{z} f^{(k+1)}(\zeta)(z-\zeta)^{k-j} d \zeta
$$

$(j=0,1, \ldots, k)$.

Each of $T_{m}^{(j)}\left(z_{m}\right)=f^{(j)}\left(z_{m}\right) \quad(j=0,1, \ldots, k)$ tends to $\infty$ as $m \rightarrow \infty$. Consider a sequence $\left(\beta_{m}\right)$ such that $\beta_{m}>0, \beta_{m} \rightarrow \infty$, and

$$
\frac{f^{(j)}\left(z_{m}\right)}{\beta_{m}} \rightarrow \infty \quad(m \rightarrow \infty) \text { for } j=0,1, \ldots, k
$$

Now we need the following statement.

Lemma. Let $Q(\zeta)$ be a polynomial of degree $l, Q\left(\zeta_{0}\right) \neq 0$; suppose that $0<$ $\beta<\left|Q\left(\zeta_{0}\right)\right|$, and let $E_{\beta}$ be the set of all points $\zeta$ such that $|Q(\zeta)| \leq \beta$. Then the visible (from the point $\zeta_{0}$ ) angular size $\varphi$ of $E_{\beta}$ satisfies the inequality

$$
\varphi \leq C \cdot l \cdot \sqrt[l]{\frac{\beta}{\left|Q\left(\zeta_{0}\right)\right|}},
$$

$C$ being an absolute constant. ( $\varphi$ is the linear Lebesgue measure of the central projection of $E_{\beta}$ to the circle $\left|\zeta-\zeta_{0}\right|=1$ from the point $\zeta_{0}$. If $l=0$, then the right-hand side of the inequality is assumed to be 0 .)

Proof of the Lemma. If $l=0$, the assertion is obvious, so let $l \geq 1$. Without loss of generality we may assume that $\zeta_{0}=0$. Let $z_{0}, z_{1}, \ldots, z_{l}$ be any $l+1$ points of $E_{\beta}$ such that $\left|\arg z_{i}-\arg z_{j}\right| \geq \alpha$ (where $0<\alpha \leq \frac{\pi}{2}$ ) when $i \neq j$. Then, obviously, $i \neq j$ implies $\left|z_{i} / z_{j}-1\right| \geq \delta=\sin \alpha \geq \frac{2}{\pi} \alpha$. Considering the equalities $a_{0} \cdot 1+a_{1} z_{j}+\cdots+a_{l} z_{j}^{l}=Q\left(z_{j}\right) \quad(j=0,1, \ldots, l)$ as a system of linear algebraic equations with respect to the coefficients $a_{0}, a_{1}, \ldots, a_{l}$ of the polynomial $Q(\zeta)\left(a_{0}=Q(0)\right)$, we have

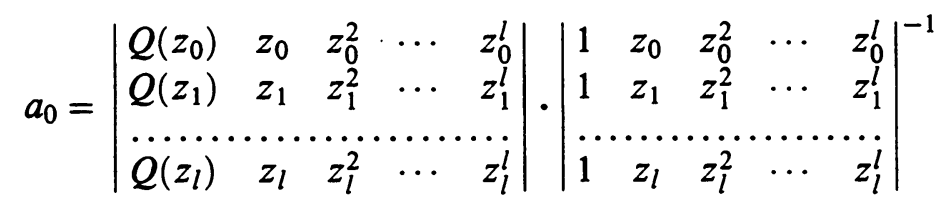

$$
\begin{aligned}
& =\left(W_{l+1}\left(z_{0}, \ldots, z_{l}\right)\right)^{-1}\left(Q\left(z_{0}\right) z_{1} z_{2} \ldots z_{l} W_{l}\left(z_{1}, \ldots, z_{l}\right)\right. \\
& -Q\left(z_{1}\right) z_{0} z_{2} \cdots z_{l} W_{l}\left(z_{0}, z_{2}, \ldots, z_{l}\right) \\
& \left.+\cdots+(-1)^{l} Q\left(z_{l}\right) z_{0} z_{1} \cdots z_{l-1} W_{l}\left(z_{0}, z_{1}, \ldots, z_{l-1}\right)\right),
\end{aligned}
$$

where $W_{*}(\cdots)$ stands for a Vandermonde determinant of degree $*$. Therefore, $a_{0}$ equals a sum whose first term is

$$
\begin{aligned}
Q\left(z_{0}\right) & \cdot z_{1} z_{2} \cdots z_{l} \cdot \frac{1}{\left(z_{1}-z_{0}\right)\left(z_{2}-z_{0}\right) \cdots\left(z_{l}-z_{0}\right)} \\
= & \frac{Q\left(z_{0}\right)}{\left(1-z_{0} / z_{1}\right)\left(1-z_{0} / z_{2}\right) \cdots\left(1-z_{0} / z_{l}\right)}
\end{aligned}
$$

evidently, the modulus of this term is not greater than $\beta / \delta^{l}$. Estimating the rest of the terms in the same way, we obtain

$$
\left|a_{0}\right| \leq(l+1) \beta / \delta^{l} \text { or } \delta \leq\left((l+1) \beta /\left|a_{0}\right|\right)^{1 / l} \text {, }
$$


which implies

$$
\alpha \leq \frac{\pi}{2} \delta \leq \pi\left(\beta /\left|a_{0}\right|\right)^{1 / l} .
$$

Therefore, for any $l+1$ points $z_{j} \in E_{\beta}(j=0,1, \ldots, l)$ there are at least two of them, say $z_{k}$ and $z_{l}(k \neq l)$, such that the angle between the segments $\left[0, z_{k}\right]$ and $\left[0, z_{l}\right]$ is not greater than $\gamma=\pi\left(\beta /\left|a_{0}\right|\right)^{1 / l}$.

Now consider all the subsets $W$ of $E_{\beta}$ such that for any two distinct points in $W$ the angular distance between them is more than $\gamma$. Evidently, among these subsets $W$ there is at least one maximal; denote it by $W^{*}$. The foregoing shows that $W^{*}$ contains $\leq l$ points, while its maximality means that any point $z \in E_{\beta}$ is at an angular distance $\leq \gamma$ from some point in $W^{*}$. Consequently, $E_{\beta}$ is contained in the union of $\leq l$ angles, the spread of each of them being equal to $2 \gamma$. The lemma is proved (with $C=2 \pi$ ).

Apply the lemma to the polynomials $T_{m}^{(j)}(\zeta)$ for $j=0,1, \ldots, k$. We have $T_{m}^{(j)}\left(z_{m}\right)=f^{(j)}\left(z_{m}\right)$; hence, by (3) and the lemma, the visible (from $z_{m}$ ) angular size of the set $X_{m}$ of all points $z$ for which at least one of the inequalities

$$
\left|T_{m}^{(j)}(z)\right| \leq \beta_{m}
$$

$(j=0,1, \ldots, k)$ holds tends to 0 as $m \rightarrow \infty$.

Thus for any sufficiently large $m$ (which guarantees also that $\left|z_{m}\right|>\frac{1}{2}$ ) there exists a ray from $z_{m}$ that intersects the circle $\left\{|z|=\frac{1}{2}\right\}$ and on which

$$
\left|T_{m}^{(j)}(z)\right|>\beta_{m} \text { for } j=0,1, \ldots, k \text {. }
$$

Let $\tilde{z}_{m}$ be the first intersection of the above ray with $\left\{|z|=\frac{1}{2}\right\}$; for $m$ large enough we have

$$
\max _{z \in\left[z_{m}, \tilde{z}_{m}\right]}\left|f^{(k+1)}(z)\right|>\beta_{m} / 2
$$

(because $\left|f^{(k)}\left(\tilde{z}_{m}\right)\right|$ and $\left|z_{m}-\tilde{z}_{m}\right|$ are bounded, while, by (3), $\left|f^{(k)}\left(z_{m}\right)\right| / \beta_{m} \rightarrow$ $\infty$ as $m \rightarrow \infty)$ and, furthermore,

$$
\left|f^{(k+1)}\left(z_{m}\right)\right|<\beta_{m} / 2 .
$$

Denote by $z_{m}^{\prime}$ the first point on $\left[z_{m}, \tilde{z}_{m}\right]$, where

$$
\left|f^{(k+1)}(z)\right|=\beta_{m} / 2 \text {. }
$$

Since

$$
\left|z_{m}-\tilde{z}_{m}\right| \leq \sqrt{1-(1 / 2)^{2}}<1,
$$

it follows from (2) that

$$
\left|R_{m}^{(j)}\left(z_{m}^{\prime}\right)\right| \leq \beta_{m} / 2 \quad(j=0,1, \ldots, k)
$$

and from (1) and (4) that

$$
\left|f^{(j)}\left(z_{m}^{\prime}\right)\right| \geq\left|T_{m}^{(j)}\left(z_{m}^{\prime}\right)\right|-\beta_{m} / 2>\beta_{m} / 2 \quad(j=0,1, \ldots, k) .
$$

Because

$$
\left|f^{(k+1)}\left(z_{m}^{\prime}\right)\right|=\beta_{m} / 2,
$$

we have

$$
f^{(j)}\left(z_{m}^{\prime}\right) \rightarrow \infty \quad(m \rightarrow \infty) \text { for } j=0,1, \ldots, k+1 .
$$


The above result may be slightly improved:

Let $\varphi(r)$ be a nondecreasing positive continuous function on $[0,1)$; suppose that for some sequence $\left(z_{n}\right)$ in $\mathbb{D}$

$$
f\left(z_{n}\right) / \varphi\left(\left|z_{n}\right|\right) \rightarrow \infty \text { as } n \rightarrow \infty .
$$

Then there exists a sequence $\left(z_{n}^{*}\right)$ in $\mathbb{D}$ such that

$$
f^{(j)}\left(z_{n}^{*}\right) / \varphi\left(\left|z_{n}^{*}\right|\right) \rightarrow \infty \quad(n \rightarrow \infty)
$$

for every $j=0,1,2, \ldots$.

The corresponding result for entire functions is the following:

Let $f(z)$ be an entire function and $\left(\varphi_{j}(r)\right)_{j=0}^{\infty}$ a sequence of nondecreasing positive continuous functions on $[0, \infty)$ such that

(i) $\lim _{r \rightarrow \infty}\left(\varphi_{j}(A r) / \varphi_{j}(r)\right)=\infty, j=0,1,2, \ldots$, for some constant $A>1$;

(ii) $r \cdot \varphi_{j+1}(r) \leq C_{j} \varphi_{j}(r)\left(r \geq r_{j}, 0<r_{j}<\infty, C_{j}>0\right), j=0,1,2, \ldots$;

(iii) for some sequence $\left(z_{n}\right)$ in $\mathbb{C}$

$$
f\left(z_{n}\right) / \varphi_{0}\left(\left|z_{n}\right|\right) \rightarrow \infty \text { as } n \rightarrow \infty .
$$

Then there exists a sequence $\left(z_{n}^{*}\right)$ in $\mathbb{C}$ such that

$$
f^{(j)}\left(z_{n}^{*}\right) / \varphi_{j}\left(\left|z_{n}^{*}\right|\right) \rightarrow \infty \quad(n \rightarrow \infty)
$$

for every $j=0,1,2, \ldots$.

We note that for any transcendental entire function, $f(z)$, there exists a sequence of functions $\left(\varphi_{j}(r)\right)_{j=0}^{\infty}$ satisfying the assumptions of this statement; hence, the latter statement (whose proof in essence coincides with the above one) provides a solution to yet another problem from [1]: to give for such $f(z)$ an elementary proof of the existence of a sequence $\left(z_{n}\right)$ in $\mathbb{C}$ for which

$$
f^{(j)}\left(z_{n}\right) \rightarrow \infty \text { as } n \rightarrow \infty
$$

$(j=0,1,2, \ldots)$.

The above estimates are far from being exact. It would be interesting to know whether the following statement is true.

Suppose that $\varphi(\cdot) \in C^{\infty}(0, R)$ (where $0<R \leq \infty$ ) and $\varphi^{(j)}(r)>0$ for all $j=0,1,2, \ldots, 0<r<R$. Let $f(z)$ be an analytic function in $D_{R}=$ $\{z:|z|<R\}$ such that

$$
\underset{r / R}{\limsup }\left(\max _{|z|=r}|f(z)| / \varphi(r)\right)=\infty .
$$

Then there exists a sequence $\left(z_{n}\right),\left|z_{n}\right| \nearrow R$, such that

$$
f^{(j)}\left(z_{n}\right) / \varphi^{(j)}\left(\left|z_{n}\right|\right) \rightarrow \infty \text { as } n \rightarrow \infty
$$

$(j=0,1,2, \ldots)$.

\section{ACKNOWLEDGMENT}

I thank the referee whose suggestions made it possible to present the proof in a more direct and clear way. 


\section{REFERENCES}

1. J. Langley and L. A. Rubel, Some problems about unbounded analytic functions, 199 Research Problems, Lecture Notes in Math., vol. 1043, Springer, New York, 1984, pp. 595596.

2. L. A. Rubel, Unbounded analytic functions and their derivatives on plane domains, Bull. Inst. Math. Acad. Sinica 12 (1984), 363-377.

C/O Lee Rubel, Department of Mathematics, 1409 West Green Street, University of ILLINOIS, URBANA, ILLINOIS 61801 